\title{
Effective trapping time of electrons and holes in different silicon materials irradiated with neutrons, protons and pions
}

\author{
G. Kramberger*, V. Cindro, I. Mandić, M. Mikuž, M. Zavrtanik \\ Jožef Stefan Institute and Department of Physics, University of Ljubljana, Jamova 39, SI-1000 Ljubljana, Slovenia
}

Received 22 January 2001; received in revised form 3 May 2001; accepted 10 May 2001

\begin{abstract}
Silicon diodes fabricated on oxygenated and non-oxygenated silicon wafers with different bulk resistivities $(1,2$ and $15 \mathrm{k} \Omega \mathrm{cm}$ ) were irradiated with neutrons, pions and protons to fluences up to $2.4 \times 10^{14} \mathrm{n} / \mathrm{cm}^{-2}$. Effective trapping times for electrons and holes were determined by the charge correction method in the temperature range between $-50^{\circ} \mathrm{C}$ and $20^{\circ} \mathrm{C}$. The measured effective trapping probabilities scale linearly with fluence and decrease with increasing temperature. Irradiation with charged hadrons resulted in about 30\% higher trapping probabilities than with neutrons at the same equivalent fluence. No dependence on silicon resistivity and oxygen concentration was found. The temperature dependence could be parameterized by a power-law scaling. Accelerated annealing at $60^{\circ} \mathrm{C}$ showed a $30 \%$ increase of hole trapping, measured at $10^{\circ} \mathrm{C}$, and a decrease by about the same amount for electron trapping, both at a time scale of 10 h. (C) 2002 Elsevier Science B.V. All rights reserved.
\end{abstract}

PACS: $85.30 . \mathrm{De} ; 29.40 . \mathrm{Wk} ; 29.40 . \mathrm{Gx}$

Keywords: Effective carrier trapping time; Silicon detectors; Charge collection efficiency

\section{Introduction}

Segmented silicon detectors will be widely used in experiments at the Large Hadron Collider (LHC) for tracking of charged particles. The high luminosity and the large proton-proton inelastic cross section will result in intense fluxes of heavy particles, mainly pions and neutrons, to which silicon detectors and accompanying read-out electronics will be exposed. As an example, the ATLAS Semiconductor Tracker will accumulate

\footnotetext{
*Corresponding author. Tel.: + 386-1-477-3512, fax: + 3861-425-7074.

E-mail address: gregor.kramberger@ijs.si (G. Kramberger).
}

fluences up to about $2 \times 10^{14} \mathrm{~cm}^{-2} 1 \mathrm{MeV}$ neutron non-ionizing energy loss (NIEL) equivalent during its ten year operation at the collider [1].

Trapping of the drifting charge in traps having emission times longer than the shaping time of the electronics $(\sim 25 \mathrm{~ns})$ will reduce the signal [2]. This could be partly overcome by over-depleting the detector, thereby reducing the drift time and probability for trapping. However, the increased effective dopant concentration and leakage current after irradiation impose practical limitations to applicable voltages due to detector breakdown and thermal runaway.

Defect dynamics and the related change of effective dopant concentration and leakage current 
with time have been extensively studied - see for example [3]. Data on trapping times are, however, relatively scarce [4,5]. The knowledge of the effective trapping time is, however, of vital importance for an accurate prediction of detector performance at the LHC.

In this work, we present a systematic measurement of electron and hole trapping times after irradiation of silicon diodes with neutrons, pions and protons to fluences up to $2.4 \times 10^{14} \mathrm{~cm}^{-2} 1 \mathrm{MeV}$ neutron NIEL equivalent. The method used for determination of the effective trapping time is based on the correction of time-resolved current pulse shapes due to trapping, recently proposed independently by two groups [6,7]. The diodes used in this study were fabricated on silicon of various resistivities and oxygen concentrations. The effective trapping times were determined in the temperature range between $-50^{\circ} \mathrm{C}$ and $20^{\circ} \mathrm{C}$. Evolution of trapping with time after irradiation was also investigated.

\section{The measurement set-up}

A transient current technique (TCT) set-up was used to measure the current response of the detectors to short light pulses. Diodes were mounted on a temperature-controlled optical liquid-nitrogen cryostat, allowing light injection from both $\mathrm{p}^{+}$and $\mathrm{n}^{+}$sides of the diode. Current signals were amplified with a fast current amplifier and transmitted to a $500 \mathrm{MHz}$ oscilloscope. A red laser with a wavelength of $670 \mathrm{~nm}$ (absorption depth $3.3 \mu \mathrm{m}$ at $20^{\circ} \mathrm{C}$ ) and pulse width of approximately $1 \mathrm{~ns}$ was used. The short penetration depth of the red light gives the possibility to inject electrons by $\mathrm{p}^{+}$side illumination and holes by $\mathrm{n}^{+}$side illumination.

The charge correction method for the determination of the effective trapping time is based on the assumption that the decrease of charge due to trapping is exponential with time, as the charge drifts through the detector. Therefore, the integral of the induced current (induced charge) does not saturate in an irradiated detector but also exhibits a rise at voltages above the full depletion voltage $\left(V_{\mathrm{FD}}\right)$. At higher voltages, the drift time is reduced due to the higher electric field and thus less charge gets trapped. To determine the effective trapping time $\left(\tau_{\text {eff }}\right)$, the measured induced currents $\left(I_{\mathrm{m}}(t)\right)$ are corrected with an exponential $\exp \left(t / \tau_{\text {eff }}\right)$ so that the integral of the corrected current gives equal charges for all voltages above $V_{\mathrm{FD}}$.

For a detailed explanation of the set-up and of the charge correction method the reader is referred to [6].

\subsection{Irradiations}

Neutron irradiations took place in the core of the TRIGA nuclear reactor of the Jožef Stefan Institute in Ljubljana. The measured and simulated spectrum of neutrons with energies ranging from thermal to fast $(\sim 10 \mathrm{MeV})$ can be found in Ref. [8]. Fast neutron $(E>0.1 \mathrm{MeV})$ fluxes up to $4 \times 10^{12} \mathrm{~cm}^{-2} \mathrm{~s}^{-1}$ were measured by gold activation [9] and scaled with the calculated damage factor. They agree with the values obtained from the leakage current method [10] to better than $10 \%$ [11]. During the irradiation, the diodes were kept at a temperature around $20^{\circ} \mathrm{C}$. The longest irradiation time was about $5 \mathrm{~min}$.

Irradiations with pions were performed at the Paul Scherrer Institute in Villigen, Switzerland. The energy of pions was $200 \mathrm{MeV}$. Equivalent fluences were determined from aluminum foil activation using the damage factor 1.2 (average of values reported in Refs. [12,13]) as well as with the leakage current method. The highest fluence of $2.4 \times 10^{14} \mathrm{~cm}^{-2}$ was reached within a day. The temperature during irradiation was around $25^{\circ} \mathrm{C}$.

Protons of $24 \mathrm{GeV} / c$ from the CERN PS were used for proton irradiations. Fluences were determined with the same methods as for pion irradiations. The damage factor used for PS protons is 0.62 [3].

\subsection{Samples}

Several $\mathrm{p}^{+}-\mathrm{n}-\mathrm{n}^{+}$pad detectors processed on high $(15 \mathrm{k} \Omega \mathrm{cm})$ and low resistivity $(1,2 \mathrm{k} \Omega \mathrm{cm})$ standard and oxygen enriched silicon wafers were irradiated. The samples were produced at ST Microelectronics and at Brookhaven National Laboratory (BNL). Their properties and treatment 
Table 1

Irradiation fluences and diode properties. The samples labeled with W were processed by ST Microelectronics on Wacker silicon wafers, while those labeled with BNL were processed on Topsil silicon. Time and temperature of diffusion oxygenation is given. The silicon crystals were cut along the $\left\langle\begin{array}{lll}1 & 1 & 1\end{array}\right\rangle$ plane

\begin{tabular}{|c|c|c|c|}
\hline Sample & Oxygenation & $\begin{array}{l}\text { Initial resistivity } \\
\text { and } V_{\mathrm{FD}}\end{array}$ & Fluences $\Phi_{\mathrm{eq}}\left(10^{13} \mathrm{n} / \mathrm{cm}^{2}\right)$ \\
\hline W339 & No & $\begin{array}{l}15 \mathrm{k} \Omega \mathrm{cm} \\
V_{\mathrm{FD}}=10-15 \mathrm{~V}\end{array}$ & $\begin{array}{l}\text { Neutrons: } 0,2.5,5,7.5,10,15,20 \\
\text { Pions: } 5.3,16.7 \\
\text { Protons: } 5.6,10.9,19.9\end{array}$ \\
\hline W336 & No & $\begin{array}{l}2 \mathrm{k} \Omega \mathrm{cm} \\
V_{\mathrm{FD}} \approx 135 \mathrm{~V}\end{array}$ & $\begin{array}{l}\text { Neutrons:- } \\
\text { Pions: } 5.3,16.7 \\
\text { Protons: } 10.9,19.9\end{array}$ \\
\hline W333 & No & $\begin{array}{l}1 \mathrm{k} \Omega \mathrm{cm} \\
V_{\mathrm{FD}} \approx 295 \mathrm{~V}\end{array}$ & $\begin{array}{l}\text { Neutrons:- } \\
\text { Pions: } 9.5,24 \\
\text { Protons: } 10.9,19.9\end{array}$ \\
\hline W317 & $\begin{array}{l}60 \mathrm{~h} \\
\text { at } \\
T=1200^{\circ} \mathrm{C}\end{array}$ & $\begin{array}{l}15 \mathrm{k} \Omega \mathrm{cm} \\
V_{\mathrm{FD}} \approx 20 \mathrm{~V}\end{array}$ & $\begin{array}{l}\text { Neutrons: } 2.5,7.5 \\
\text { Pions:- } \\
\text { Protons: } 5.6,19.9\end{array}$ \\
\hline W309 & $\begin{array}{l}60 \mathrm{~h} \\
\text { at } \\
T=1200^{\circ} \mathrm{C}\end{array}$ & $\begin{array}{l}2 \mathrm{k} \Omega \mathrm{cm} \\
V_{\mathrm{FD}} \approx 135 \mathrm{~V}\end{array}$ & $\begin{array}{l}\text { Neutrons:- } \\
\text { Pions: } 9.5,24 \\
\text { Protons: } 10.9,19.9\end{array}$ \\
\hline W301 & $\begin{array}{l}60 \mathrm{~h} \\
\text { at } \\
T=1200^{\circ} \mathrm{C}\end{array}$ & $\begin{array}{l}1 \mathrm{k} \Omega \mathrm{cm} \\
V_{\mathrm{FD}} \approx 295 \mathrm{~V}\end{array}$ & $\begin{array}{l}\text { Neutrons:- } \\
\text { Pions: } 9.5,24 \\
\text { Protons: } 10.9\end{array}$ \\
\hline BNL920 & No & $\begin{array}{l}\approx 1 \mathrm{k} \Omega \mathrm{cm} \\
V_{\mathrm{FD}}=170-210 \mathrm{~V}\end{array}$ & $\begin{array}{l}\text { Neutrons: } 10,20 \\
\text { Pions:- } \\
\text { Protons:- }\end{array}$ \\
\hline BNL917 & $\begin{array}{l}12 \mathrm{~h} \\
\text { at } \\
T=1200^{\circ} \mathrm{C}\end{array}$ & $\begin{array}{l}\approx 1 \mathrm{k} \Omega \mathrm{cm} \\
V_{\mathrm{FD}} \approx 250 \mathrm{~V}\end{array}$ & $\begin{array}{l}\text { Neutrons: } 10,20 \\
\text { Pions:- } \\
\text { Protons:- }\end{array}$ \\
\hline
\end{tabular}

are listed in Table 1. A hole $(\varnothing=2 \mathrm{~mm})$ in the metalization on the $\mathrm{p}^{+}$side of the diode was used for light injection, while on the $\mathrm{n}^{+}$side a mesh metalization was provided for the same purpose.

During and after irradiation the diodes were kept unbiased. After the irradiation they were mounted onto an aluminum support using a roomtemperature curing conductive glue. Then they were annealed at $T=20^{\circ} \mathrm{C}$ to the minimum in $V_{\mathrm{FD}}$. The evolution of $V_{\mathrm{FD}}$ during annealing was measured with the $C-V$ method at the frequency $v=10 \mathrm{kHz}$. The minimum values of $V_{\mathrm{FD}}$ are shown in Fig. 1. There is a nearly perfect agreement of $V_{\mathrm{FD}}$ for samples fabricated on nonoxygenated high-resistivity material at equal NIEL equivalent fluences for different particles. Taking into account the negligible remaining donor concentration after irradiation, this confirms the dosimetry measurements at different irradiation sites. Once on reaching the minimum in $V_{\mathrm{FD}}$, the diodes were stored at $T=-17^{\circ} \mathrm{C}$ until the measurements of effective trapping times were performed. 

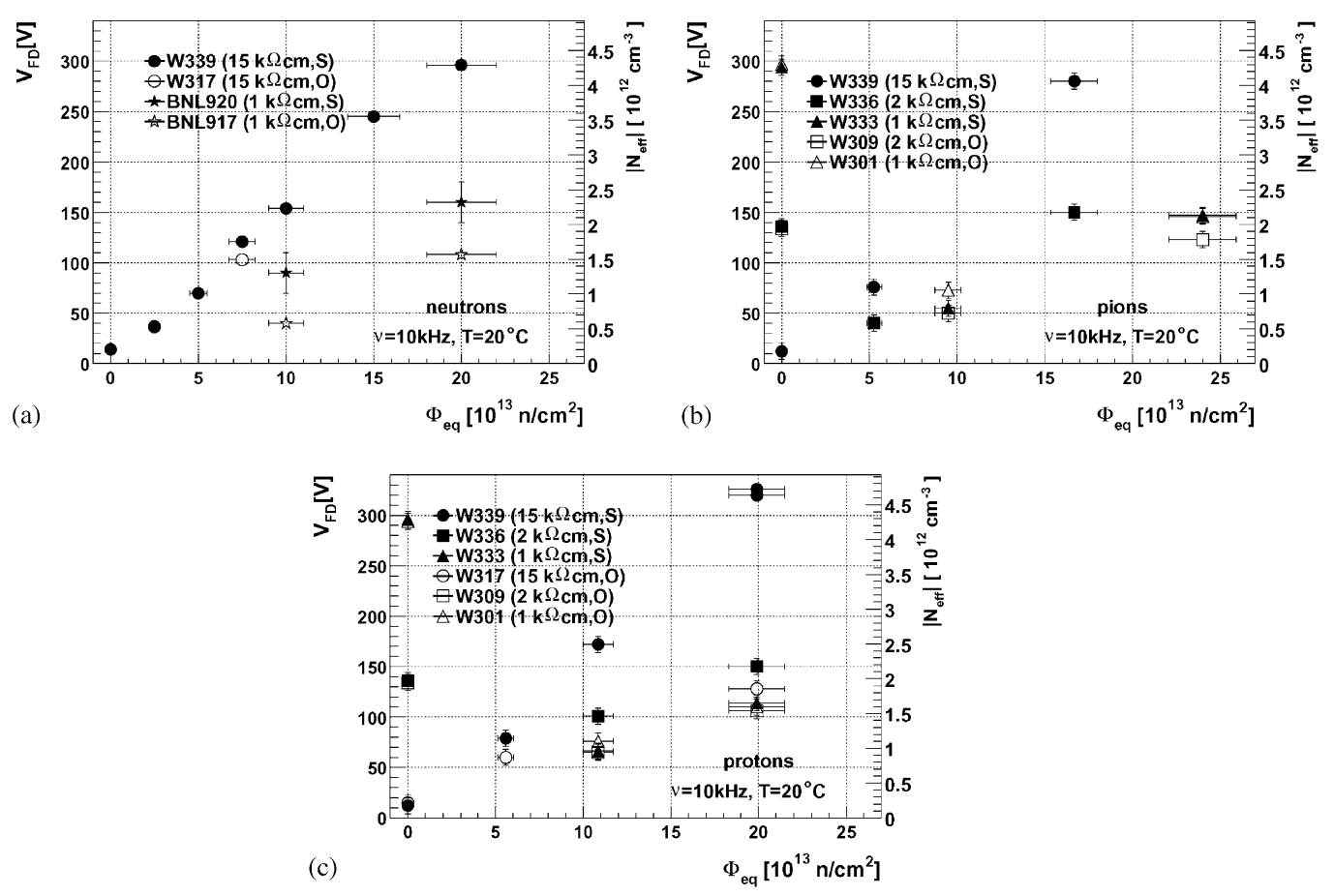

Fig. 1. $V_{\mathrm{FD}}$ as measured with the $C-V$ method at the annealing minimum: (a) neutron, (b) pion and (c) proton irradiation. $V_{\mathrm{FD}}$ of the BNL920 samples has a larger uncertainty due to non-ideal $C-V$ characteristics. The material type is given in brackets: $\mathrm{O}$ for oxygenated and $\mathrm{S}$ for standard material.

\section{Results}

If the thermal velocity $v_{\text {th }}$ is large compared to the drift velocity (which applies to our case), the integrated path length $l$ of the charge $q$, and thus the number of collisions, is proportional to the drift time and thermal velocity $\left(l=v_{\text {th }} t\right)$, that allows to introduce the effective trapping probability by

$q(t)=q(t=0) \mathrm{e}^{-t / \tau_{\mathrm{eff}}}$.

Here, $q(t)$ represents the drifting charge, electrons or holes, created at $t=0$. The trapping probability can be expressed as

$\frac{1}{\tau_{\mathrm{eff}, \mathrm{h}}}=\sum_{t} N_{t}\left(1-P_{t}^{\mathrm{e}, \mathrm{h}}\right) \sigma_{t_{\mathrm{e}, \mathrm{h}}} v_{\mathrm{th}_{\mathrm{e}, \mathrm{h}}}$.

Here, $N_{t}$ denotes the trap concentration, $P_{t}^{\mathrm{e}, \mathrm{h}}$ its occupation probability with the relevant carrier, $v_{\mathrm{th}_{\mathrm{e}, \mathrm{h}}}$ the thermal velocity of drifting carriers and $\sigma_{t_{\mathrm{e}, \mathrm{h}}}$ the carrier capture cross-section. The sum runs over all traps. For traps introduced directly by irradiation, $N_{t}$ has the form

$N_{t}=g_{t} \Phi_{\text {eq }} f_{t}(t)$

with $g_{t}$ the introduction rate and $f_{t}(t)$ representing (exponential) trap annealing with time after irradiation. On the other hand, Eq. (3) with appropriately modified $f_{t}(t)$ can also describe traps originating from decays of primary defects. A more general form, including $\Phi_{\mathrm{eq}}^{2}$ terms, would be needed to describe traps formed by reactions between defects.

If traps described by Eq. (3) $\left(N_{t} \propto \Phi_{\text {eq }}\right)$ dominate, the effective trapping probability at a given temperature and time after irradiation can be parameterized as

$\frac{1}{\tau_{\mathrm{eff}}, \mathrm{h}}=\beta_{\mathrm{e}, \mathrm{h}}(t, T) \Phi_{\mathrm{eq}}$. 
The aim of this study is to evaluate $\beta_{\mathrm{e}, \mathrm{h}}$ as a function of irradiation particle type, silicon material and temperature, and its dependence on time after irradiation.

\subsection{Material and particle dependence}

The effective trapping probability for electrons and holes, measured at $T=-10^{\circ} \mathrm{C}$ after $\sim 10$ days of room temperature annealing, for different particles and silicon materials can be seen in Fig. 2. A clear linear dependence of the trapping probability on $\Phi_{\text {eq }}$ can be observed, indicating an effective absence of trapping centers formed in second-order reactions. The resistivity of the silicon material in the range from 1 to $15 \mathrm{k} \Omega \mathrm{cm}$ does not influence the effective trapping probability. There is also no difference between standard
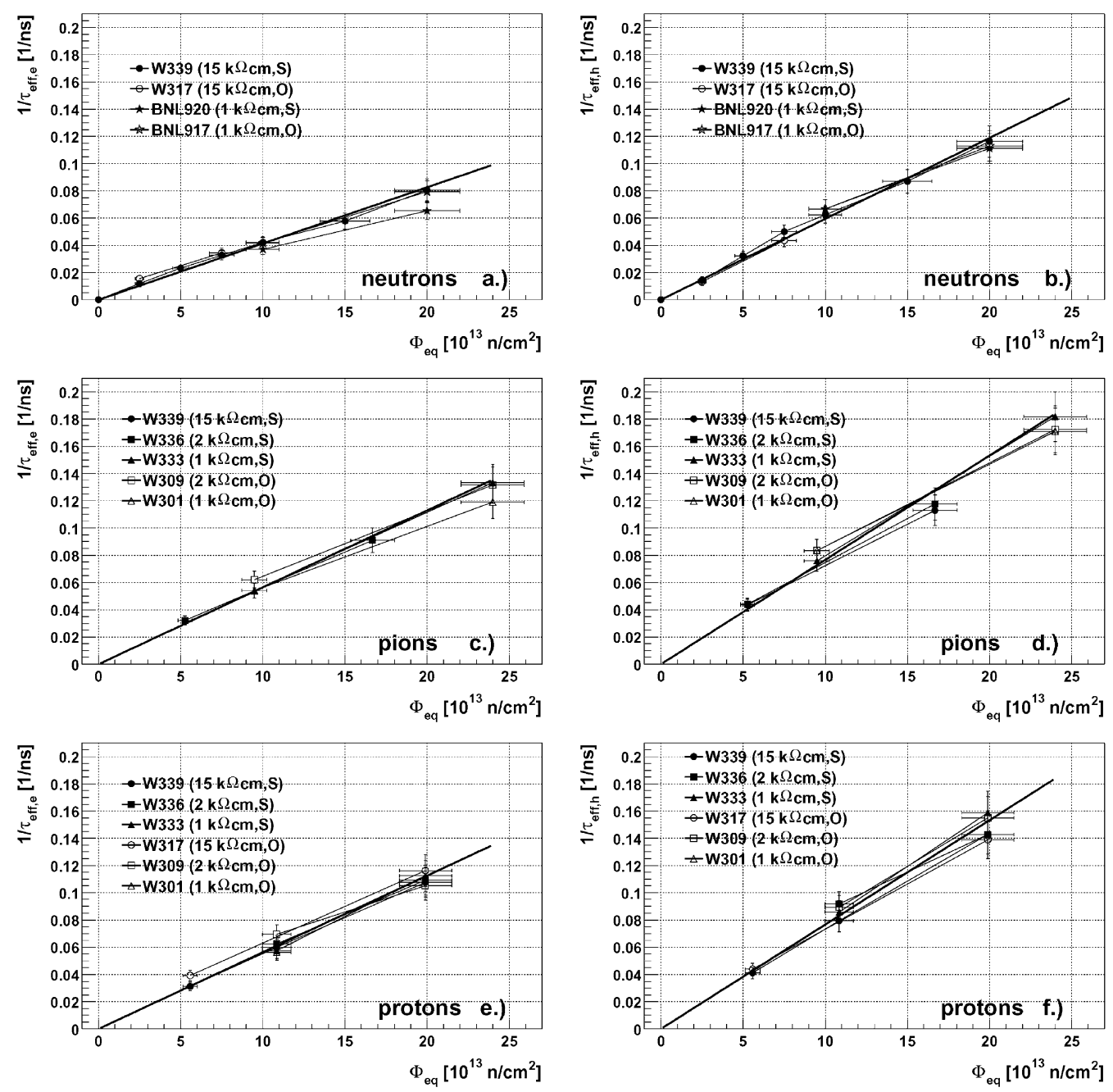

Fig. 2. Fluence dependence of effective trapping probability for electrons and holes for neutron, pion and proton-irradiated samples: (a) electrons (neutron irradiation), (b) holes (neutron irradiation), (c) electrons (pion irradiation), and (d) holes (pion irradiation), (e) electrons (proton irradiation) and (f) holes (proton irradiation). Measurements were taken at $T=-10^{\circ} \mathrm{C}$ after $\sim 10$ days of annealing at room temperature. Resistivity and material type are given in brackets: O-oxygenated and S-standard material. 
Table 2

$\beta$ for electrons and holes for different particle types at $T=$ $-10^{\circ} \mathrm{C}$ after $\sim 10$ days of room temperature annealing. The error observed does not include a $\sim 10 \%$ systematic error on dosimetry

\begin{tabular}{lll}
\hline & $\beta_{\mathrm{e}}\left(10^{-16} \mathrm{~cm}^{2} / \mathrm{ns}\right)$ & $\beta_{\mathrm{h}}\left(10^{-16} \mathrm{~cm}^{2} / \mathrm{ns}\right)$ \\
\hline Neutrons & $4.1 \pm 0.1$ & $6.0 \pm 0.2$ \\
Pions & $5.7 \pm 0.2$ & $7.7 \pm 0.2$ \\
Protons & $5.6 \pm 0.2$ & $7.7 \pm 0.2$ \\
\hline
\end{tabular}

and oxygenated silicon materials, although the latter exhibits a smaller increase of negative space charge upon charged hadron irradiation [3].

It appears, however, that different particle types have different $\beta_{\mathrm{e}, \mathrm{h}}$ as can be seen in Table 2. $\beta_{\mathrm{e}, \mathrm{h}}$ for charged hadrons are about $30-40 \%$ larger than those for neutrons. The effective trapping probability thus does not scale with NIEL when comparing neutrons with charged particles. As demonstrated in Section 2.1, the agreement of $V_{\mathrm{FD}}$ of standard material at the same equivalent fluences for different irradiations excludes a dosimetry error that is required to cause the observed level of $\beta_{\mathrm{e}, \mathrm{h}}$ dependence on particle type. A possible qualitative explanation could be that NIEL from charged hadrons produces more point defects than NIEL of neutrons which in turn creates more cluster defects. Point defects thus appear to be more efficient as trapping centers. Since oxygen acts as a vacancy sink and is observed so as not to influence trapping, these defects are likely to be interstitial-related.

\subsection{Temperature dependence}

Three parameters in $\beta_{\mathrm{e}, \mathrm{h}}$ depend on temperature: thermal velocity of the carriers, capture crosssections and occupation probabilities of the traps. A square-root temperature dependence is characteristic for $v_{\mathrm{th}_{\mathrm{e}, \mathrm{h}}}$. The capture cross-section dependence on temperature is poorly known, scaling as $\sigma(T) / \sigma\left(T_{0}\right)=\left(T / T_{0}\right)^{m}$ with $m$ ranging between -2 and 2 as suggested in Refs. [14,15].

Let us assume that one defect dominates trapping of the drifting electrons and equivalently, there exists a dominating hole trap. The occupa- tion probability of a trap, described by the energy level $E_{t}$, in the depleted region can be calculated using Shockley-Read-Hall statistics as [16] follows:

$$
\begin{aligned}
& P_{t}^{\mathrm{e}}(T)=\frac{1}{\left(c_{\mathrm{e}} / c_{\mathrm{h}}\right) \chi_{t}^{2}+1}, \quad P_{t}^{\mathrm{h}}(T)=1-P_{t}^{\mathrm{e}}(T), \\
& p \approx n \approx 0 \\
& \chi_{t}=\exp \left(\frac{E_{t}-E_{i}}{k_{\mathrm{B}} T}\right), \quad c_{\mathrm{e}, \mathrm{h}}=\sigma_{t_{\mathrm{e}, \mathrm{h}}} v_{\mathrm{th}_{\mathrm{e}, \mathrm{h}}} \\
& E_{i}=\frac{E_{\mathrm{g}}}{2}+\frac{3}{4} k_{\mathrm{B}} T \ln \left(\frac{P_{\mathrm{v}}}{N_{\mathrm{c}}}\right), \quad \frac{P_{\mathrm{v}}}{N_{\mathrm{c}}}=0.371 \\
& E_{\mathrm{g}}=1.12 \mathrm{eV}
\end{aligned}
$$

where $E_{i}$ denotes the Fermi level in intrinsic silicon, $k_{\mathrm{B}}$ the Boltzmann constant, and $P_{\mathrm{v}}$ and $N_{\mathrm{c}}$ the effective densities of states in the valence and conduction band, respectively.

Temperature scaling of $\beta_{\mathrm{e}, \mathrm{h}}$ dominated by a single trap can thus be written as

$$
\begin{aligned}
\beta_{\mathrm{e}, \mathrm{h}}(T) & =\frac{1}{\tau_{\mathrm{eff}_{\mathrm{e}, \mathrm{h}}} \Phi_{\mathrm{eq}}} \\
& =\beta_{\mathrm{e}, \mathrm{h}}\left(T_{0}\right) \frac{1-P_{t}^{\mathrm{e}, \mathrm{h}}(T)}{1-P_{t}^{\mathrm{e}, \mathrm{h}}\left(T_{o}\right)}\left(\frac{T}{T_{0}}\right)^{m+1 / 2} .
\end{aligned}
$$

In order to study the dependence of effective trapping probability on temperature, measurements for all the diodes from Table 1 were performed in the temperature interval from 223 to $293 \mathrm{~K}$. The temperature range was constrained by practical limitations. For lower temperatures, fast drift, especially of electrons, rendered the experimental determination of $\tau_{\text {eff }}$ questionable. And at higher temperatures, the resulting reverse current was too high to be properly handled by our experimental set-up.

The measured temperature dependence of average $\beta_{\mathrm{e}, \mathrm{h}}$ for all three particle types is shown in Fig. 3 exhibiting a $20-30 \%$ decrease of trapping probability over the temperature range covered. An examination of terms in Eq. (8) showed that such behavior could be caused either by a negative $m$ in cross-section scaling, or by a near to mid-gap position of the trap, supported by an appropriate $c_{\mathrm{e}} / c_{\mathrm{h}}$ ratio, or a combination of the two effects. 

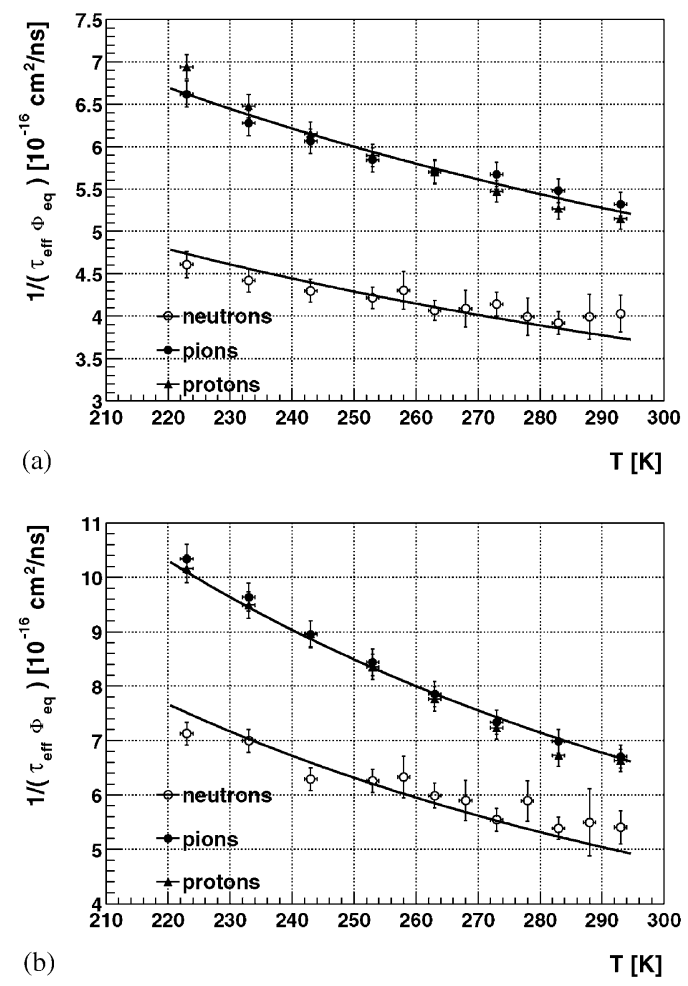

Fig. 3. Temperature dependence of $\beta$ for: (a) electrons and (b) holes. The measured points are the average of $\beta$ 's for all measured samples.

Table 3

Parameters $\kappa_{\mathrm{e}, \mathrm{h}}$, determining temperature scaling of $\beta_{\mathrm{e}, \mathrm{h}}$, as obtained from the fit of Eq. (9) to the measured data

\begin{tabular}{ll}
\hline$\kappa_{\mathrm{e}}=-0.86 \pm 0.06$ & $\kappa_{\mathrm{h}}=-1.52 \pm 0.07$ \\
\hline
\end{tabular}

Therefore, an extraction of separate trap parameters was considered unjustified. An effective parameterization of the form

$\beta_{\mathrm{e}, \mathrm{h}}(T)=\beta_{\mathrm{e}, \mathrm{h}}\left(T_{0}\right)\left(\frac{T}{T_{0}}\right)^{\kappa_{\mathrm{e}, \mathrm{h}}}$

with $\kappa_{\mathrm{e}, \mathrm{h}}$ universal for all particle types yielded acceptable fits (see Fig. 3) to all data. Fit results are listed in Table 3. This could indicate, that the same two traps, albeit introduced at different rates, play the dominant role in

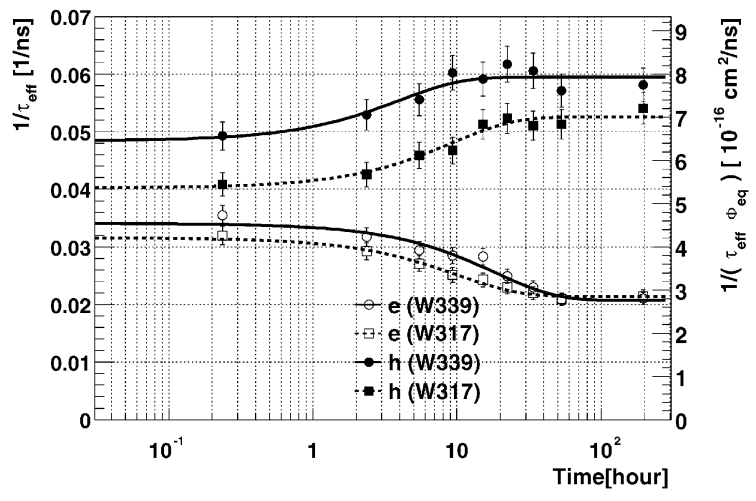

Fig. 4. Annealing of effective trapping probability at $60^{\circ} \mathrm{C}$. Measurements were taken at $T=10^{\circ} \mathrm{C}$.

trapping induced by neutron and charged hadron irradiation.

\subsection{Annealing of effective trapping probability}

With time, defects can either decay or interact with others to form new ones. The consequence of this is a change in effective trapping probability, covered by the annealing function $f_{t}(t)$ in Eq. (3). Annealing of $\beta_{\mathrm{e}, \mathrm{h}}$ was studied with two diodes from wafers $\mathrm{W} 339$ (standard, $15 \mathrm{k} \Omega \mathrm{cm}$ ) and W317 (oxygenated, $15 \mathrm{k} \Omega \mathrm{cm}$ ) irradiated with neutrons to $7.5 \times 10^{13} \mathrm{~cm}^{-2}$. After the diodes reached the minimum in $V_{\mathrm{FD}}$ at room temperature, accelerated annealing was performed in steps at $60^{\circ} \mathrm{C}$. Between annealing steps TCT measurements were taken at $T=10^{\circ} \mathrm{C}$. The diodes were mounted on to the same Peltier temperature regulator to ensure equal temperature history. Dependence of $\beta_{\mathrm{e}, \mathrm{h}}$ on annealing time is shown in Fig. 4. It can be seen that, for both standard and oxygenated material, $\beta_{\mathrm{e}}$ at $10^{\circ} \mathrm{C}$ decreases with time after irradiation by about $35 \%$, while $\beta_{\mathrm{h}}$ increases by about $30 \%$. An elementary model assuming the decay of the dominant electron trap into another stable one was evaluated and observed to describe the annealing data correctly. The same model, applied to the dominant hole trap, also fits the time dependence of $\beta_{\mathrm{h}}$. The relevant fit function was

$\beta_{\mathrm{e}, \mathrm{h}}(t)=\beta_{0_{\mathrm{e}, \mathrm{h}}} \mathrm{e}^{-t / \tau_{\mathrm{e}, \mathrm{h}}}+\beta_{\infty_{\mathrm{e}, \mathrm{h}}}\left(1-\mathrm{e}^{-t / \tau_{\mathrm{e}, \mathrm{h}}}\right)$ 
Table 4

Amplitudes and time constants for the parameterization of $\beta$ annealing

\begin{tabular}{|c|c|c|c|c|c|c|}
\hline & $\begin{array}{l}\beta_{0_{\mathrm{e}}} \\
\left(10^{-16} \mathrm{~cm}^{2} / \mathrm{ns}\right)\end{array}$ & $\begin{array}{l}\beta_{\infty_{\mathrm{e}}} \\
\left(10^{-16} \mathrm{~cm}^{2} / \mathrm{ns}\right)\end{array}$ & $\begin{array}{l}\beta_{0_{\mathrm{h}}} \\
\left(10^{-16} \mathrm{~cm}^{2} / \mathrm{ns}\right)\end{array}$ & $\begin{array}{l}\beta_{\infty \mathrm{h}} \\
\left(10^{-16} \mathrm{~cm}^{2} / \mathrm{ns}\right)\end{array}$ & $\begin{array}{l}\tau_{\mathrm{e}} \\
(\mathrm{h})\end{array}$ & $\begin{array}{l}\tau_{\mathrm{h}} \\
(\mathrm{h})\end{array}$ \\
\hline W339 & $4.5 \pm 0.2$ & $2.8 \pm 0.1$ & $6.5 \pm 0.3$ & $7.9 \pm 0.2$ & $19 \pm 5$ & $4.2 \pm 2.3$ \\
\hline W317 & $4.2 \pm 0.2$ & $2.8 \pm 0.1$ & $5.4 \pm 0.3$ & $7.0 \pm 0.2$ & $10.6 \pm 3.5$ & $9.7 \pm 4.5$ \\
\hline
\end{tabular}

Table 5

Temperature scaling parameter $\kappa_{\mathrm{e}, \mathrm{h}}$ as obtained from the fit of Eq. (9) to temperature dependence of the effective trapping probability for diodes W339 and W317 at the end of annealing

\begin{tabular}{lll}
\hline & $\kappa_{\mathrm{e}}$ & $\kappa_{\mathrm{h}}$ \\
\hline W339 & $-1.55 \pm 0.24$ & $-1.50 \pm 0.24$ \\
W317 & $-1.58 \pm 0.20$ & $-1.50 \pm 0.20$ \\
\hline
\end{tabular}

with $\beta_{0_{\mathrm{e}, \mathrm{h}}} \equiv \beta_{\mathrm{e}, \mathrm{h}}$ and $\beta_{\infty_{\mathrm{e}, \mathrm{h}}}$ the trapping rates at early and late annealing times, respectively.

The results of the fits are gathered in Table 4. The same time development would also be obtained by assuming a trap with $\beta_{\mathrm{s}}=\beta_{\infty}$, stable throughout the annealing, and decay or creation of a second defect with $\beta_{\mathrm{d}}=\beta_{0}-\beta_{\infty}$. Parameters for standard and oxygenated samples exhibit the same behavior within the rather large uncertainties. Annealing times are of order of $10 \mathrm{~h}$ at $60^{\circ} \mathrm{C}$, about the same order of magnitude as characteristic times of reverse annealing. Although depending largely on the activation energy, the effect of trapping probability annealing can thus be expected to play a rather marginal role in the lifetime of a typical LHC experiment.

After the last annealing step, temperature scans of both diodes were performed. The temperature scaling parameter $\kappa_{\mathrm{e}, \mathrm{h}}$ (Table 5) of the effective trapping probability was found to be comparable with its value in the minimum of $V_{\mathrm{FD}}$ (Table 3) for holes, while for electrons its value increased nearly by a factor of two. As a consequence, $\beta_{\mathrm{e}}$ at the end of annealing turned out to be larger than $\beta_{\mathrm{e}}$ at early annealing times, if measured at temperatures lower than $-40^{\circ} \mathrm{C}$ (Fig. 5).

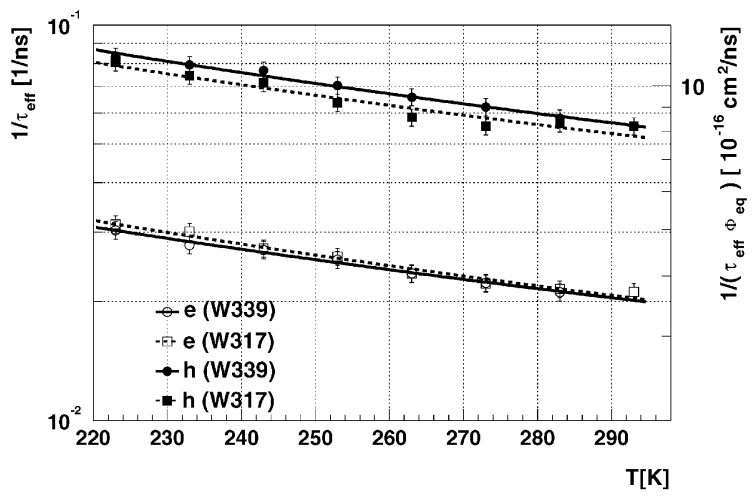

Fig. 5. Temperature dependence of the effective trapping probability for the W317 and W339 samples after the annealing procedure.

\section{Conclusions}

Several sets of diodes of different resistivities and oxygen content were irradiated with neutrons, pions and protons up to $\Phi_{\text {eq }}=2.4 \times 10^{14} \mathrm{~cm}^{-2}$. TCT measurements were performed as the irradiated diodes annealed to the minimum in $V_{\mathrm{FD}}$ at room temperature. Using the charge correction method the electron and hole effective trapping probabilities were determined for all diodes in the temperature range between 223 and $293 \mathrm{~K}$.

The effective trapping probability was found to scale linearly with fluence and not to depend on oxygenation level and resistivity. Holes were observed to have higher trapping probability than electrons. It is seen that charge trapping does not scale according to the NIEL hypothesis since charged hadrons induce $30-40 \%$ more trapping than neutrons at the same NIEL equivalent fluence. 
For both electrons and holes the effective trapping probability decreases with temperature by $20-30 \%$ over the investigated -50 to $+20^{\circ} \mathrm{C}$ temperature range. A power scaling law, universal in terms of particle type, was found to parameterize the data well.

Distinct annealing behavior of effective trapping probability for electrons and holes was observed. The electron effective trapping probability decreases by $\sim 35 \%$, while the hole effective trapping probability increases by $\sim 30 \%$. Time constants for these changes are of the order of $10 \mathrm{~h}$ at $60^{\circ} \mathrm{C}$.

\section{Acknowledgements}

The authors would like to thank B. Dezillie and $\mathrm{Z}$. Li for providing the BNL samples and to the ROSE collaboration, especially M. Moll, for the STM samples. Special thanks are due to M. Glaser for his assistance with pion and proton irradiations.

\section{References}

[1] ATLAS Collaboration: Inner Detector Technical Design Report, Vols. I and II, CERN/LHCC/97-16,17, 1997.

[2] V. Cindro, et al., Nucl. Instr. and Meth. A 439 (2000) 337.

[3] RD48 Collaboration: G. Lindström, et al., Radiation hard silicon detectors-developments by the RD48 (ROSE) Collaboration, presented at fourth STD Hiroshima
Conference, Hiroshima, Japan, March 23-25, 2000, ROSE/TN/2000-03, Nucl. Instr. and Meth. A 466 (2001) 308.

[4] H.W. Kraner, et al., Nucl. Instr. and Meth. A 326 (1993) 350 .

[5] S. Martí i García, et al., A model of charge collection for irradiated $\mathrm{p}^{+} \mathrm{n}$ detectors, presented at Ninth International Workshop on Vertex Detectors, Sleeping Bear Dunes National Lakeshore, Michigan, USA, September 10-15, 2000, Nucl. Instr. and Meth. A 473 (2001) 128.

[6] G. Kramberger, et al., Determination of effective trapping time of electrons and holes in irradiated silicon, presented at Third International Conference on Radiation Effects in Semiconductor Materials, Detectors and Devices, Firenze, June 2000 (ATL-INDET-2000-017), Nucl. Instr. and Meth. A 476 (2002) 645.

[7] T.J. Brodbeck, et al., Nucl. Instr. and Meth. A 455 (2000) 645.

[8] D. Žontar, et al., Nucl. Instr. and Meth. A 426 (1999) 51.

[9] E.S. Krištof, Characterization of neutron flux in the exposure channel F19 of the TRIGA mark II reactor in Ljubljana, Proceedings of Nuclear Energy in Central Europe '98, Terme Čatež, Slovenia, September 7-10, 1998, p. 43.

[10] M. Moll, et al., Nucl. Instr. and Meth. A 426 (1999) 87.

[11] V. Cindro, et al., Nucl. Instr. and Meth. A 450 (2000) 290.

[12] E. Fretwurst, et al., Relation between microscopic defects and macroscopic changes in silicon detector properties after hadron irradiation, Report given at First International Workshop on Defect Engineering of Advanced Semiconductor Devices Santorini, Greece, April 21-22, 1999.

[13] A. Ruzin, Nucl. Instr. and Meth. A 447 (2000) 116.

[14] V.N. Abakumov, et al., Sov. Phys. Semicond. 12 (1978) 1.

[15] A. Hallén, et al., J. Appl. Phys. 79 (1996) 3906.

[16] G. Lutz, Nucl. Instr. and Meth. A 377 (1996) 234. 